\title{
DESIGN OF HIGH PERFORMANCE DOUBLE TAIL COMPARATOR
}

\author{
Saranya P.M, Jyothish Chandran G and Shilpa K.S \\ Department of Electronics and Communication Engineering, Saintgits College of Engineering, India
}

\begin{abstract}
Comparator is an important building blocks used in analog-to-digital converters. Its function is to compare two analog inputs and delivers a logic value at the output. In this project an analysis on the delay of various dynamic comparators are presented. Based on the analysis a new dynamic comparator is designed for fast operations. Positive feedback mechanism is used to regenerate the analog input signal into full scale digital level. This design is a modification of conventional double-tail comparator. Addition of a few transistors to the conventional double-tail comparator results in remarkably reduced time delay. Kick-back noise of this comparator is also reduced. The large voltage variations in the internal nodes are coupled to the input nodes, which will disturb the input nodes-this is called kick-back noise. This is reduced by inserting switches before the input transistors of comparator. The performance of conventional comparator and proposed comparator circuits are evaluated based on Cadence $180 \mathrm{~nm}$ CMOS process models.
\end{abstract}

Keywords:

Analog to Digital Converter (ADC), Double Tail Comparator, Kick Back Noise

\section{INTRODUCTION}

Comparison is one of the basic operations used in analog to digital comparator. In the conversion process, input signal is sampled first. This sampled signal is then applied to the comparators to find the digital equivalent of the input analog value. Comparators give a logical value, which determine whether one input is higher or lower than the other one. The selection of an $\mathrm{ADC}$ is defined by the requirements: if we need speed, then use a fast $\mathrm{ADC}$, if we want precision, use an accurate ADC, or if we are constrained in space, use a compact ADC.

Comparators produce a logical value, which indicates whether one input is higher or lower than the other one [8]. Nowadays, the demand for high speed ADCs is increasing. Since, comparators are the basic building block used in ADCs we require comparators with high speed, less delay and less kick-back noise. Kick-back noise is one of the major problems affecting the comparator and it will affect the accuracy of the analog-to-digital converter. Many techniques are available to reduce this issue.

Usually, dynamic comparators are preferred in ADCs because these comparators have less delay and less power consumption [7]. Positive feedback mechanism is provided by back-to-back inverters which converts a smaller voltage difference to full scaledigital level output.

When supply voltage is small, design of high-speed comparator becomes complex. A voltage boosting method is presented in [5] and this method is apt for CMOS design containing transistors with threshold voltage comparable to the supply voltage. Here, during comparison phase supply voltage is locally boosted to higher levels. Time delay of the comparator is reduced by liming the voltage swing of first stage to $V_{d d} / 2$ in [12]. Some extra circuits are inserted to the conventional dynamic comparator to enhance the performance in [4] and [6]. A doubletail latch type comparator, which has high input impedance and full swing output, is proposed in [2].

Performance of a comparator can also explain in terms of the accuracy it provides. Uncertainty in the transition region of output is mainly due to noise.

This paper is divided into six sections, first section is the introduction. Section 2 is the literature review of this topic. Section 3 gives a brief idea about conventional dynamic comparators. Proposed double-tail comparator is presented in section 4. Simulation results are given in section 5. Finally, conclusions are drawn in section 6.

\section{DYNAMIC COMPARATORS}

Comparison of inputs is done at distinct time points in the case of dynamic comparators. These comparators require a clock or triggered signal. Dynamic comparators uses positive feedback to enhance the gain and have to make decisions in a limited time slot and. The working of dynamic comparators is divided in to two stages: reset phase and comparison phase.

\subsection{CONVENTIONAL DYNAMIC COMPARATOR}

Conventional dynamic comparator is a simple comparator used in ADCs since it has high input impedance, rail-to-rail output swing and no static power consumption. The schematic diagram of the conventional dynamic comparator is shown in the Fig1. The operation of this comparator can be divided in to two phases: reset phase and comparison phase.

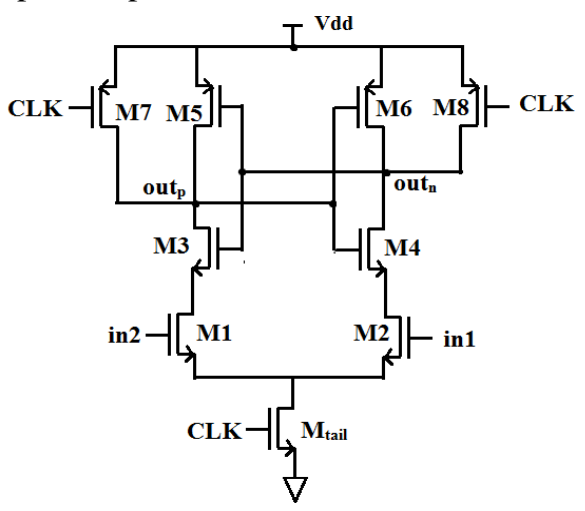

Fig.1. Schematic diagram of conventional dynamic comparator

During reset phase $C L K$ is at $0 \mathrm{~V}$ and during comparison phase, $C L K$ is at $V_{d d}$. Inputs $i n_{1}$ and $i n_{2}$ are applied to the input transistors $M_{1}$ and $M_{2}$. In the reset phase, transistor $M_{\text {tail }}$ is off. But, transistors $M_{5}$ and $M_{6}$ start conducting in reset phase and which pull up both the outputs out $_{p}$ and $\mathrm{out}_{n}$ to $V_{d d}$ to have a valid logic state during 
reset. Then, as the time passes, the precharged outputs starts to discharge to ground depending on the inputs applied.

Assume $i n_{1}>i n_{2}$, then out $_{n}$ will discharge faster than $o u t_{p}$. Whenever, out $t_{n}$ falls below $V_{d d^{-}} V_{t h n}$ before out $_{p}$, the corresponding pmos transistor $M_{5}$ start conducting and initiate regeneration. Finally, $o u t_{p}$ will be pull back to $V_{d d}$ and $o u t_{n}$ discharges to ground. The reverse will be happen if $i n_{1}<i n_{2}$.

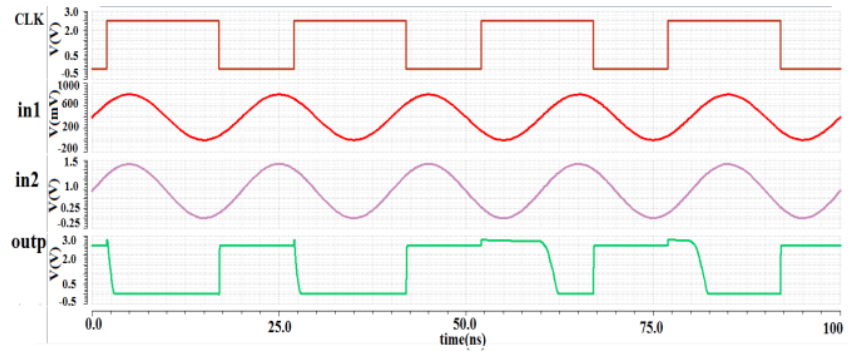

Fig.2. Simulation result of conventional dynamic comparator

Some problems exist in conventional comparator even though this structure has high input impedance and rail-to-rail output swing. First disadvantage is that, a sufficiently high supply voltage is needed for the proper working of the circuit due to several stacked transistors. There is only one current path via $M_{\text {tail }}$ which contributes the current for both input stage and regeneration stage, which is the next problem. Because, each stage has its own current requirements like, input stage requires small current and regeneration stage requires large current to have fast regeneration. Transient simulation of design is shown in Fig.2.

\subsection{CONVENTIONAL DOUBLE TAIL DYNAMIC COMPARATOR}

The schematic diagram of the conventional double-tail is shown in Fig.3. This design has less stacking of transistors and therefore can be used at low supply voltages, which is the advantage of this topology.

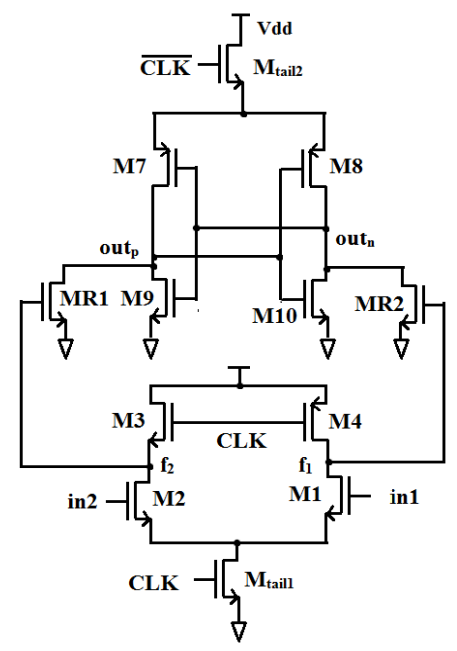

Fig.3. Schematic diagram of conventional double-tail comparator

In order to provide separate current path for regeneration path, one extra tail transistor is added to the circuit. Two intermediate transistors are indicated by $M R_{1}$ and $M R_{2}$ and intermediate points are denoted by $f_{1}$ and $f_{2}$. During reset phase, both tail transistors $M_{\text {tail } 1}$ and $M_{\text {tail } 2}$ are off. The intermediate points are pulled to $V_{d d}$ by both $M_{3}$ and $M_{4}$ transistors. Then intermediate transistors turn on and which will cause both the outputs to discharge to ground. In the comparison phase, both tail transistors turn on and intermediate point voltage starts discharging depending upon the inputs applied. If $i n_{1}>i n_{2}, f_{1}$ voltage discharge faster than $f_{2}$ voltage. So, out $t_{p}$ will be pull up to $V_{d d}$ through $M_{7}$. Finally, out $t_{p}$ will be at logic high level. At the end of comparison stage both the intermediate point voltages discharges to ground, which is the main drawback of this circuit is that. So, these nodes have to be charged from ground to $V_{d d}$ in the next reset phase, which may lead to increased power consumption.

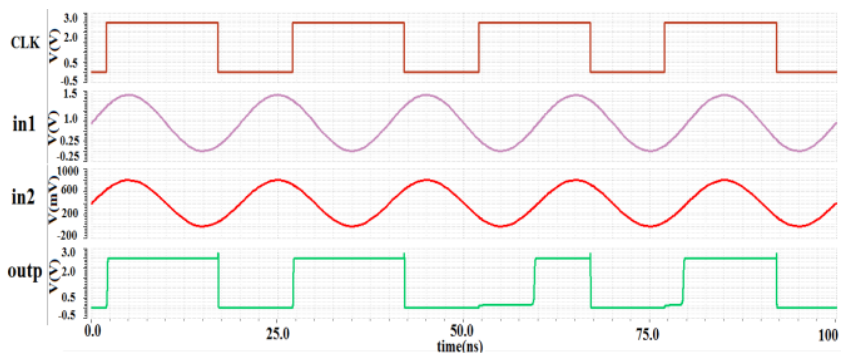

Fig.4. Simulation result of conventional double-tail comparator

By analyzing the conventional double-tail comparator, it can be concluded that delay of this circuit is inversely proportional to the difference in the input voltage $\left(\Delta V_{i n 1 / i n 2}\right)$. Difference in input voltage and difference in intermediate point voltage $\left(\Delta V_{f 1 / f 2}\right)$ are directly proportional. Hence increasing this difference would reduce the delay of the circuit. Transient simulation of the design is shown in Fig.4.

This double-tail comparator is much better than conventional one in terms of delay. But both the circuits are affected by kickback noise problem. In latched comparators, the voltage variations on the output nodes may couple to the input nodes through parasitic capacitances. So, the input is disturbed, which may degrade the accuracy of the converter. This disturbance is called kick-back noise. Many techniques are available to reduce this noise in comparator circuits.

\section{PROPOSED DOUBLE TAIL COMPARATOR}

The Proposed double-tail comparator without noise reduction is demonstrated in Fig.5. The Proposed double-tail comparator with noise reduction technique is demonstrated in Fig.7. As explained in the previous section, it requires increasing intermediate point voltage $\left(\Delta V_{f 1 / f 2}\right)$ to reduce delay.

\subsection{PROPOSED COMPARATOR WITHOUT NOISE REDUCTION}

In order to increase the difference in intermediate point voltage, two control transistors $M C_{1}$ and $M C_{2}$ are added to the intermediate point in a cross-coupled manner [1].

Operation of the proposed comparator is as follows. During reset phase $(C L K=0)$, both tail transistors are off. The $f_{1}$ and $f_{2}$ voltages are pulled up to $V_{d d}$ through $M_{3}$ and $M_{4}$. In the comparison phase $\left(C L K=V_{d d}\right)$, intermediate point voltages start to discharge. Suppose $i n_{1}>i n_{2}$, then $f_{1}$ discharge faster than $f_{2}$. As 
$f_{1}$ continues discharging, the corresponding control transistor $\left(M C_{1}\right)$ turns on, which will pull-up out $t_{p}$ back to $V_{d d}$.

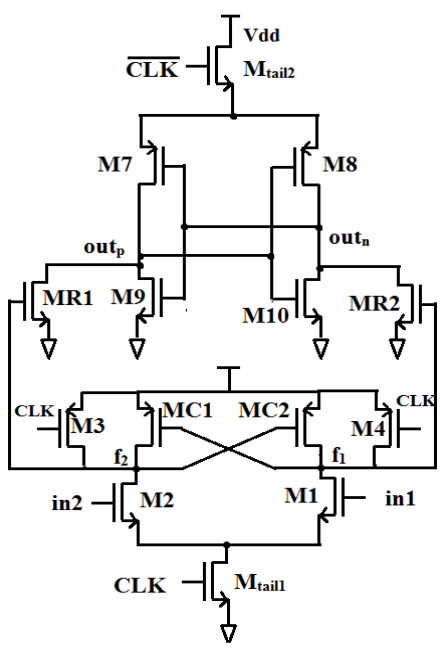

Fig.5. Schematic diagram of proposed comparator without noise reduction

This will cause the complete discharging of $f_{1}$ to ground. Finally $o u t_{p}$ will be at $V_{d d}$ after comparison. Advantage of this circuit is that during the reset stage, it is not needed to pull both $f_{1}$ and $f_{2}$ back to $V_{d d}$ since, one of the points have already been pulled to $V_{d d}$ in the previous comparison phase itself. Transient simulation of this design is shown in Fig.6.

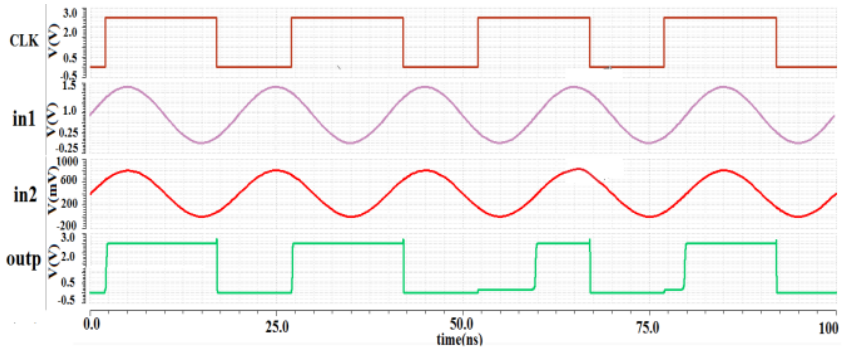

Fig.6. Simulation result of proposed comparator without noise reduction

\subsection{PROPOSED COMPARATOR WITH NOISE REDUCTION}

Kick-back noise is one of the major problems in comparators because; it may affect the precision of analog-to-digital converters. Many techniques are available to reduce this noise. Drains of the comparator are isolated from the output nodes using switches to eliminate the disturbances in [9]. But, disturbances arise during comparison phase also. Neutralization technique is introduced in [10] and [11] in which capacitors having equal magnitude as that of parasitic capacitance are added to the circuit. But, this technique also cannot achieve a significant noise reduction.

The Fig.7 shows the proposed comparator with kick-back reduction technique. This method can be used in any latched comparator [3]. Noise can be reduced by preventing any disturbances on the input nodes coupled from the output points. This is achieved by inserting switches before the input transistors of comparator.

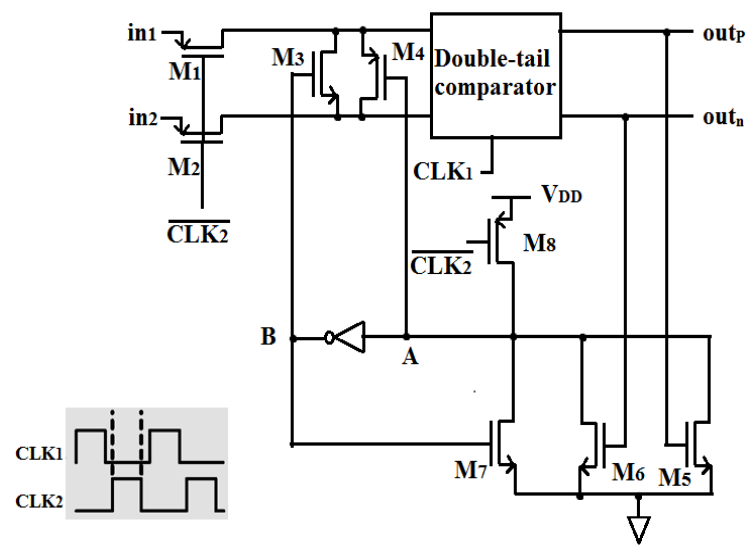

Fig.7. Schematic diagram of proposed comparator with noise reduction

In Fig.7, the box represents the proposed comparator circuit without noise reduction. Transistors $M_{1}$ and $M_{2}$ represents the control transistors. The $M_{3}$ and $M_{4}$ are the reset transistors. The $C L K_{1}$ is used to synchronize the operation of comparator and $C L K_{2}$ synchronizes operation of noise reduction circuit. When $C L K_{1}=0\left(C L K_{2}=V_{d d}\right)$, comparator is in reset phase. Both input switches are on. Node $A$ is pulled to $V_{d d}$ through $M_{8}$ transistor and hence node $B$ is at $0 \mathrm{~V}$. So, both reset transistors are off. Comparator reset both its outputs to logic low level. At the end of $C L K_{2}, M_{1}$ and $M_{2}$ turn off, hence preventing any disturbances from the output on the input points during regeneration.

During regeneration $\left(C L K_{1}=V_{d d}\right.$ and $\left.C L K_{2}=0\right)$, one of the two inputs will be pulled to high level, which will turn on $M_{5}$ or $M_{6}$ and hence pull down node $A$ to ground. Finally both the reset transistors $M_{3}$ and $M_{4}$ turn on and reset the inputs. With this method, kick-back noise in the comparator circuit can be eliminated to a large extend.

\section{SIMULATION RESULTS}

In order to compare the proposed comparator with other conventional comparators, all the circuits have been simulated in $180 \mathrm{~nm}$ CMOS technology with $V_{d d}=2.5 \mathrm{~V}$. The transient simulation of proposed circuit with noise reduction technique is shown in Fig.8.

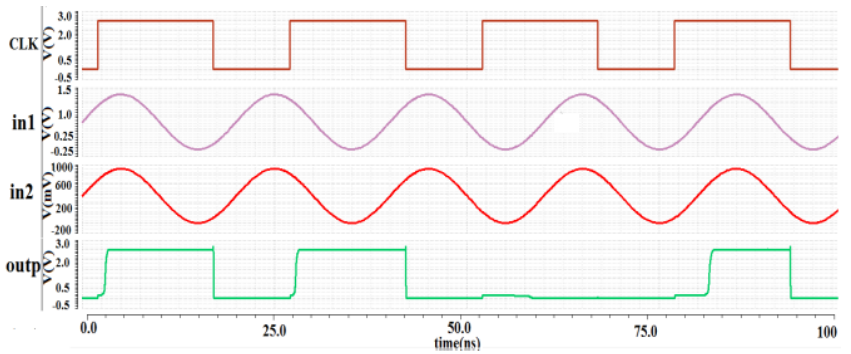

Fig.8. Simulation result of proposed comparator with noise reduction

The Table. 1 shows the delay and kick-back noise of various circuit types like conventional comparator, conventional doubletail comparator, and proposed comparator without noise reduction technique and with noise reduction technique. 
Table.1. Comparison of delay and noise of comparators

\begin{tabular}{|c|c|c|}
\hline Circuit Type & $\begin{array}{c}\text { Delay } \\
\text { (in ps) }\end{array}$ & $\begin{array}{c}\text { Kick-Back } \\
\text { Noise } \\
\text { (in mV) }\end{array}$ \\
\hline Conventional comparator & 285.46 & 404.5 \\
\hline $\begin{array}{c}\text { Conventional double-tail } \\
\text { comparator }\end{array}$ & 105.32 & 301.7 \\
\hline $\begin{array}{c}\text { Proposed double-tail comparator } \\
\text { without noise reduction }\end{array}$ & 75.51 & 299.3 \\
\hline $\begin{array}{c}\text { Proposed double-tail comparator } \\
\text { with noise reduction }\end{array}$ & 80.27 & 95.3 \\
\hline
\end{tabular}

It is clear from the table that the delay of conventional comparator was about 285.46ps. Delay is reduced to $105.32 \mathrm{ps}$ for conventional double-tail comparator. This is because there are two tail transistors, one of which provides enough current for the input section and the other transistor provides enough current for the regeneration section. The main advantage of this circuit is that it has reduced stacking effect. So, all the transistors will get enough current for the operations and delay is reduced.

In the proposed comparator the delay is about $75.51 \mathrm{ps}$ and $80.27 \mathrm{ps}$. This is because of the two control transistors added. These will help in increasing the difference in intermediate voltage as time passes. Delay is inversely proportional to the difference in intermediate voltage and hence reduced the delay.

Kick-back noise of the conventional comparator was about $404.5 \mathrm{mV}$. It is reduced by an amount of $300 \mathrm{mV}$ to $95 \mathrm{mV}$. This reduction is achieved by adding two switches at the input, which will be opened during comparison phase so that feedback from output to the input can be reduced.

\section{CONCLUSION}

In this paper an analysis on the delay of dynamic comparators is presented. Conventional dynamic comparator and conventional dynamic comparator were analyzed initially. Then from the conclusions, a new double-tail comparator with less delay was proposed. Kick-back noise reduction technique is also included in this design. Simulation results in 180nm CMOS technology shows that this design considerably reduces the kick-back noise of the comparator. Other existing noise reduction techniques do not solve the problem significantly. On analysis using Cadence tool kit, the proposed circuit is able to reduce the delay by 200ns and kick-back noise by an amount of $300 \mathrm{mV}$. As a future work, the offset of this comparator can be improved using some other techniques.

\section{REFERENCES}

[1] Samanesh Babayan-Mashhadi and Reza Lotfi, "Analysis and Design of a Low-Voltage-Power Double-Tail
Comparator", IEEE Transactions on Very Large Scale Integration Systems, Vol. 22, No. 2, pp. 343-352, 2014.

[2] D. Shinkel, E. Mensink, E. Klumperink, E. Van Tuiji and B. Nauta, "A Double-Tail Latch-Type Voltage Sense Amplifier with 18ps Setup+Hold Time", Proceedings of IEEE International Solid-State Circuits Conference, pp. 314-315, 2007.

[3] Pedro M. Figueiredo and Joao C. Vital, "Kick-back Noise Reduction Techniques for CMOS Latched Comparators", IEEE Transactions on Circuits and Systems-II: Express Briefs, Vol. 53, No. 7, pp. 541-545, 2006.

[4] B. Goll and H. Zimmermann, "A $0.12 \mu \mathrm{m}$ CMOS Comparator requiring $0.5 \mathrm{~V}$ at $600 \mathrm{MHz}$ and $1.5 \mathrm{~V}$ at $6 \mathrm{GHz}$ ", Proceedings of IEEE International Solid-State Circuits Conference, pp. 316-317, 2007.

[5] A. Mesgarani, M.N. Alam, F.Z. Nelson and S.U. Ay, "Supply Boosting Techniques for Designing very LowVoltage Mixed-Signal Circuits in Standard CMOS", Proceedings of $53^{\text {rd }}$ IEEE International Midwest Symposium on Circuits and Systems, pp. 893-896, 2010.

[6] B. Goll and H. Zimmermann, "A 65nm CMOS Comparator with modified latch to achieve $7 \mathrm{GHz} / 1.3 \mathrm{~mW}$ at $1.2 \mathrm{~V}$ and $700 \mathrm{MHz} / 47 \mu \mathrm{W}$ at $0.6 \mathrm{~V}$ ", Proceedings of IEEE International Solid-State Circuits Conference, pp. 328-329, 2009.

[7] B. Goll and H. Zimmermann, "A Comparator with Reduced Delay Time in 65-nm CMOS for Supply Voltages Down to 0.65V", IEEE Transactions on Circuits and Systems-II: Express Briefs, Vol. 56, No. 11, pp. 810-814, 2009.

[8] Jun He, Sanyi Zhan, Degang Chen and Randall L. Geiger, "Analyses of Static and Dynamic Random Offset Voltages in Dynamic Comparatos", IEEE Transactions on Circuits and Systems-I: Regular papers, Vol. 56, No. 5, pp. 911-919, 2009

[9] P. Amaral, J. Goes, N. Paulino and A. Steiger-Garcao, "An Improved Low-Voltage Low-Power CMOS Comparator to be used in High-Speed Pipeline ADCs", Proceedings of IEEE International Symposium on Circuits and Systems, pp. 141-144, 2002.

[10] Y. Wang and B. Razavi, "An 8-bit 150-MHz CMOS A/D Converter", IEEE Journal of Solid-State Circuits, Vol. 35, No. 3, pp. 308-317, 2000.

[11] L.Y. Nathawad, R. Urata, B.A. Wooley and D.A.B. Miller, "A 40-GHz-Bandwidth, 4-bit, Time-Interleaved A/D Converter using Photoconductive Sampling”, IEEE Journal of Solid-State Circuits, Vol. 38, No. 12, pp. 2021-2030, 2000.

[12] Ata Khorami and Mohammad Sharifkhani, "A High-Speed Method of Dynamic Comparators for SAR Analog to Digital Converters", Proceedings of IEEE 59 ${ }^{\text {th }}$ International Midwest Symposium on Circuits and Systems, pp. 1-4, 2016. 